\title{
Differential Short-Term Plasticity of PV and SST Neurons Accounts for Adaptation and Facilitation of Cortical Neurons to Auditory Tones
}

\author{
${ }^{\circledR}$ Michael J. Seay, ${ }^{1}{ }^{\circledR}$ Ryan G. Natan, ${ }^{2}{ }^{\circledR}$ Maria N. Geffen, ${ }^{3}$ and ${ }^{\circledR}$ Dean V. Buonomano ${ }^{1}$ \\ ${ }^{1}$ Integrative Center for Learning and Memory, Departments of Neurobiology and Psychology, University of California, Los Angeles, Los Angeles, \\ California 90095, ${ }^{2}$ Department of Molecular and Cell Biology, University of California, Berkeley, Berkeley, California 94720, and ${ }^{3}$ Department of \\ Otorhinolaryngology Head and Neck Surgery, Perelman School of Medicine, University of Pennsylvania, Philadelphia, Pennsylvania 19104
}

Cortical responses to sensory stimuli are strongly modulated by temporal context. One of the best studied examples of such modulation is sensory adaptation. We first show that in response to repeated tones pyramidal (Pyr) neurons in male mouse auditory cortex (A1) exhibit facilitating and stable responses, in addition to adapting responses. To examine the potential mechanisms underlying these distinct temporal profiles, we developed a reduced spiking model of sensory cortical circuits that incorporated the signature short-term synaptic plasticity (STP) profiles of the inhibitory parvalbumin (PV) and somatostatin (SST) interneurons. The model accounted for all three temporal response profiles as the result of dynamic changes in excitatory/inhibitory balance produced by STP, primarily through shifts in the relative latency of Pyr and inhibitory neurons. Transition between the three response profiles was possible by changing the strength of the inhibitory PV $\rightarrow \mathrm{Pyr}$ and SST $\rightarrow$ Pyr synapses. The model predicted that a unit's latency would be related to its temporal profile. Consistent with this prediction, the latency of stable units was significantly shorter than that of adapting and facilitating units. Furthermore, because of the history-dependence of STP the model generated a paradoxical prediction: that inactivation of inhibitory neurons during one tone would decrease the response of A1 neurons to a subsequent tone. Indeed, we observed that optogenetic inactivation of $\mathrm{PV}$ neurons during one tone counterintuitively decreased the spiking of $\mathrm{Pyr}$ neurons to a subsequent tone $400 \mathrm{~ms}$ later. These results provide evidence that STP is critical to temporal context-dependent responses in the sensory cortex.

Key words: adaptation; auditory cortex; parvalbumin; short-term synaptic plasticity; somatostatin; temporal

Significance Statement

Our perception of speech and music depends strongly on temporal context, i.e., the significance of a stimulus depends on the preceding stimuli. Complementary neural mechanisms are needed to sometimes ignore repetitive stimuli (e.g., the tic of a clock) or detect meaningful repetition (e.g., consecutive tones in Morse code). We modeled a neural circuit that accounts for diverse experimentally-observed response profiles in auditory cortex (A1) neurons, based on known forms of short-term synaptic plasticity (STP). Whether the simulated circuit reduced, maintained, or enhanced its response to repeated tones depended on the relative dominance of two different types of inhibitory cells. The model made novel predictions that were experimentally validated. Results define an important role for STP in temporal context-dependent perception.

Received Mar. 18, 2020; revised Sep. 16, 2020; accepted Oct. 14, 2020.

Author contributions: M.J.S., R.G.N., M.N.G., and D.V.B. designed research; M.J.S. and R.G.N. performed research; M.J.S. analyzed data; M.J.S. and D.V.B. wrote the paper.

This research was supported by the National Institutes of Health (NIH) National Institute of Mental Health Grant R01MH060163 (to D.V.B.), NIH National Institute on Deafness and Other Communication Disorders Grants 01DC015527 and R01DC014479 (to M.N.G.), and the NIH National Institute of Neurological Disorders and Stroke Grant R01NS113241 (to M.N.G.). We thank Vishwa Goudar for assistance with this project.

The authors declare no competing financial interests.

Correspondence should be addressed to Dean V. Buonomano at dbuono@ucla.edu.

https://doi.org/10.1523/JNEUROSCI.0686-20.2020

Copyright $\odot 2020$ the authors

\section{Introduction}

Auditory processing requires identification of temporal structure of stimuli on the subsecond time scale, including order, duration, interval, and temporal context. Speech, for example, is characterized not only by its spectral structure but its temporal structure, including the order and duration of phonemes as well as intervals between them (Doupe and Kuhl, 1999; Saberi and Perrott, 1999; Tallal, 2004). Despite the importance of identifying the temporal structure of auditory stimuli, the neural correlates and mechanisms of this process remain poorly understood. 
One of the best studied examples of the modulation of the responses of auditory neurons by temporal context is sensory adaptation, in which responses to pairs or trains of consecutive tones progressively decrease (Phillips et al., 1989; Shu et al., 1993; Brosch and Schreiner, 1997, 2000; Wehr and Zador, 2005; Nelken and Chechik, 2007; Rennaker et al., 2007; Sadagopan and Wang, 2009; Klampfl et al., 2012; Natan et al., 2017). Sensory adaptation is observed in the somatosensory and visual modalities as well (Ohzawa et al., 1982; Wilson, 2000; Ahissar et al., 2001; Chung et al., 2002; Katz et al., 2006; Kohn, 2007; Gutnisky and Dragoi, 2008). There is, however, significant diversity in the modulation of cortical responses by consecutive stimuli, including neurons that exhibit enhanced responses to specific spatiotemporal patterns (Brosch and Schreiner, 1997, 2000; Kilgard and Merzenich, 2002; Nelken and Chechik, 2007; Sadagopan and Wang, 2009; Phillips et al., 2017a). The diversity of adapting and facilitating responses presumably reflects the cross-purpose need to habituate to repetitive stimuli that carry little information (e. g., the tic of a clock), and to detect critical information carried by events occurring late in a sequence (e.g., in Morse code the letter $\mathrm{I}$ is represented by two consecutive dots, and the letter $\mathrm{H}$ by four dots; Klump and Gerhardt, 1987; Rose et al., 2011; Solomon and Kohn, 2014).

Rodent studies have focused primarily on adapting (i.e., decreasing) responses to sequences of tones and proposed a number of potential underlying mechanisms including shortterm synaptic depression and long-lasting inhibition (Wehr and Zador, 2005; Rennaker et al., 2007; Solomon and Kohn, 2014). More recent studies have suggested differential roles for PV and SST neurons in sensory adaptation (Natan et al., 2015, 2017) and forward suppression measured with tone pairs (Phillips et al., 2017a,b). Auditory cortical neurons, however, exhibit a range of temporal profiles in response to sequences of tones, including stable responses and progressive facilitation. The mechanisms responsible for this diversity of temporal profiles are not understood, and it remains an open question whether the adapting, stable, and facilitating responses can be explained by the same set of mechanisms or rely on fundamentally different properties.

Previous research has suggested that STP plays an important role in governing sensitivity to temporal context (Buonomano, 2000; Fortune and Rose, 2001; Chung et al., 2002; Oswald and Reyes, 2008; Rosenbaum et al., 2012; Motanis et al., 2018). Here, we characterized the different temporal profiles of auditory neurons to sequences of tones and developed a spiking neuron model that incorporates known differential short-term synaptic dynamics of PV and SST. In contrast to previous firing rate models (Natan et al., 2015; Phillips et al., 2017b), here, we use a spike-based model that accurately captures STP dynamics and the relative timing between excitation and inhibition, and makes experimental predictions about spike latency. We found that both adaptation and facilitation can be explained in terms of differences in the relative balance of inhibition originating from PV and SST neurons. The model generated a number of predictions including: (1) Different temporal profiles should be correlated with specific firing latency signatures, and (2) inactivating inhibitory neurons should prevent normally occurring STP of IPSPs, and thus alter pyramidal (Pyr) responses to subsequent tones. Both predictions were tested, and supported by the experimental evidence.

\section{Materials and Methods}

In vivo electrophysiology

The experimental data set used here was the same as that used in a previously published dataset (Natan et al., 2017). Eleven adult male PV-Cre (B6;129P2-Pvalbtm1(cre)Arbr/J) and SST-Cre (Ssttm2.1(cre)Zjh/J) mice aged 12-15 weeks were anesthetized, and AAV encoding Cre-dependent ArchT (Natan et al., 2017) was injected into auditory cortex (A1) two to four weeks before experimental recordings. During electrophysiology recording session mice were anesthetized, and a linear silicon 32-electrode probe placed into A1. Online monitoring of sound-evoked responses was used to ensure correct electrode positioning and to identify a 1.3 octave range of pure tone frequencies between 1 and $80 \mathrm{kHz}$ that evoked multiunit responses ranging from maximal to weak. Offline spike sorting was performed using commercial software.

Acoustic tone stimuli were $100-\mathrm{ms}$ tone pips separated by $300 \mathrm{~ms}$ of silence (400-ms stimulus-onset-asynchrony). Trains of eight tones of a single frequency were presented, separated by $2.4 \mathrm{~s}$ of silence. Each train used one of 10 frequencies within the 1.3 octave range in a pseudorandom and counterbalanced manner. In half of the trials, an optic fiber was used to direct 532-nm laser light into A1 from $100 \mathrm{~ms}$ before to $150 \mathrm{~ms}$ after each of four time periods: during the first tone, during the last tone, or during the silent period $400 \mathrm{~ms}$ before or $400 \mathrm{~ms}$ after the train.

Analysis of A1 recordings

Temporal profiles to the presentation of eight tones of the same frequency were classified as adapting, stable, or facilitating, by regressing the number of tone-evoked spikes against the corresponding serial position. Statistical tests (sign-rank, rank sum, or linear regression, see below) were performed using single-trial spike counts within the 10- to 70-ms period following tone onset as the outcome variable, with the significance level $\alpha$ set to 0.05 . Adapting temporal profiles were defined by a significant evoked response to the first tone and a significant negative slope across serial positions. Facilitating profiles were defined by a significant evoked response to the final tone and a significant positive regression slope. Steady temporal profiles were defined as those with a significant response to both the first and final tones of a train and a nonsignificant regression. As in previous studies, analyses were based on neuron-frequency pairs, i.e., each of the 10 frequencies used in the tone trains was tested and analyzed separately for each neuron (Natan et al., 2017).

To help ensure that our analyses only included Pyr cells, we took advantage of the Cre-dependent ArchT expression in the following manner. For PV-Cre mice, adapting and steady unit-frequencies were excluded if they had significantly lower first-tone evoked firing rate during light compared with non-light. For SST-Cre mice, steady and facilitating unit-frequencies were excluded if they had significantly lower eighth-tone evoked firing rate during light compared with non-light. Although indirect light-driven decreases in activity in Pyr cells could be produced through disinhibition (e.g., in a $\mathrm{SOM} \rightarrow \mathrm{PV} \rightarrow \mathrm{Pyr}$ ) this method decreases the likelihood of false-positive Pyr units in our analyses. Furthermore, the number of units that exhibited a light-driven decrease in activity was small: 22 adapting unit-frequencies (3.8\%), 30 steady unit-frequencies (3.6\%), and three facilitating unit-frequencies $(3.5 \%)$ were excluded in this manner.

Poststimulus time histograms (PSTHs) were calculated by convolving spike times with a Gaussian kernel of SD $10 \mathrm{~ms}$. When averaged across neuron-frequencies, PSTHs for each neuron-frequency were converted to a normalized deviation from baseline firing rate in the following manner. First, we calculated the mean and SD of the firing rate in the baseline period during the 1-s period between trials. Next, we subtracted the mean from the PSTH and divided by the SD of the baseline firing rate. Finally, we normalized each PSTH such that its maximum was 1.

For the latency analysis, PSTHs were calculated in the standard manner by summing spikes within 4-ms time bins. A neuron-frequency's tone-evoked latency was operationalized as the center of the time bin with the highest firing rate between 10 and $100 \mathrm{~ms}$ following tone onset for either the first (adapting and steady profiles) or last tone (facilitating profiles). To test for the effect of prior light on the evoked firing rate at serial position 2 , sign-rank tests were employed.

\section{Computational model}

Units were simulated as conductance-based integrate-and-fire units (Table 1). Three distinct types of neuronal units were implemented: excitatory Pyr, fast-spiking inhibitory parvalbumin (PV), and low-threshold 
Table 1. Global parameters

\begin{tabular}{lc}
\hline Parameter & Value \\
\hline Integration time step $(\mathrm{ms})$ & 0.05 \\
Temperature $\left({ }^{\circ} \mathrm{C}\right)$ & 36 \\
Cell length $(\mathrm{cm})$ & 10 \\
Cell diameter $(\mathrm{cm})$ & 10 \\
Resting membrane potential $(\mathrm{mV})$ & -65 \\
Leak conductance $\left(\mu \mathrm{S} / \mathrm{cm}^{2}\right)$ & 100 \\
Internal resistance $\left(\Omega \mathrm{s}^{2} \mathrm{~cm}\right)$ & 35 \\
Refractory period $(\mathrm{ms})$ & 3 \\
AHP reversal potential $(\mathrm{mV})$ & -80 \\
Dendritic compartment length $(\mathrm{cm})$ & 100 \\
Dendritic compartment diameter $(\mathrm{cm})$ & 0.5 \\
Noise current $(\mathrm{nA})$ & 0.005 \\
\hline
\end{tabular}

Global NEURON parameters used for all units and simulations.

inhibitory somatostatin (SST) units. The three unit types were modeled with distinct intrinsic parameters including membrane time constant, spike threshold, and an afterhyperpolarization current designed to reproduce their firing properties and spike-adaptation (see Table 2). Pyr units were modeled as two-compartment (soma and dendrite) units, while both types of inhibitory units were simulated as a single compartment unit. Simulations were implemented in the NEURON simulation environment (Hines and Carnevale, 1997), and were based on previously published models (Carvalho and Buonomano, 2009; Goudar and Buonomano, 2015). Each unit's membrane potential was subject to leak, afterhyperpolarization, and synaptic currents as follows:

$$
C_{m} \frac{d V}{d t}=g_{L}\left(V_{m}-E_{L}\right)+g_{A H P}\left(V_{m}-E_{A H P}\right)+g_{s y n}\left(V_{m}-E_{s y n}\right) .
$$

Excitatory and inhibitory synaptic transmission was modeled using $\alpha$ synapses with forward and backward binding (Destexhe et al., 1994). Short-term synaptic plasticity (STP) was incorporated at all synapses using the Tsodyks-Markram formulation (Markram et al., 1998; Carvalho and Buonomano, 2011), wherein repeated presynaptic spikes modulated the maximal synaptic conductance by a product of resources $(R)$ and availability $(u)$ :

$$
\begin{gathered}
R_{n+1}=1-\left[1-R_{\mathrm{n}}\left(1-u_{n}\right)\right] \cdot e^{-\frac{\Delta t_{s p}}{\tau_{d}}} ; \\
u_{n+1}=U+u_{n}(1-U) \cdot e^{-\frac{\Delta t_{s p}}{\tau_{f}}},
\end{gathered}
$$

where $\tau_{\mathrm{d}}$ and $\tau_{\mathrm{f}}$ are the time constants of depression and facilitation respectively, and $U$ can be interpreted as the initial release probability. $R$ and $u$ are updated at the time of each presynaptic spike, and $\Delta t_{s p}$ is the interval between the current and previous spike. All cellular and synaptic parameters were defined in consultation with the NeuroElectro project and studies of paired patch-clamp recordings from connected cortical cells (Reyes et al., 1998; Reyes and Sakmann, 1999; Beierlein et al., 2003; Tan et al., 2008; Levy and Reyes, 2011, 2012; Takesian et al., 2013). Synaptic parameters are shown in Table 3.

Each unit received three distinct input fibers, and tones were simulated as a single spike in each input fiber once in sequence with a fixed 5$\mathrm{ms}$ interspike interval (simulations with interspike intervals in the 5- to 10 - ms range and the same weight parameters yielded similar results). Optogenetic inactivation was modeled by transiently injecting a current of $-0.01 \mathrm{nA}$ to the inactivated unit.

The simulated circuit was based on the feedforward connectivity of a cortical microcircuit (Douglas and Martin, 2007; Cardin, 2018), and thus did not incorporate the positive feedback between Pyr neurons, or negative feedback produced by Pyr to inhibitory neuron activation. This simplifying assumption was made because we focused primarily on the short-latency responses of A1 neurons in anesthetized mice, which are
Table 2. Unit parameters

\begin{tabular}{lccc}
\hline Parameter & Pyr & PV & SST \\
\hline Membrane time constant $(\mathrm{ms})$ & 15 & 7.5 & 19 \\
Spike threshold $(\mathrm{mV})$ & -35 & -40 & -45 \\
Spike duration $(\mathrm{ms})$ & 1 & 0.5 & 0.75 \\
Membrane capacitance $\left(\mu \mathrm{F} / \mathrm{cm}^{2}\right)$ & 1.5 & 0.75 & 1.9 \\
AHP conductance increment $\left(\mu \mathrm{S} / \mathrm{cm}^{2} / \mathrm{spike}\right)$ & 100 & 25 & 50 \\
AHP decay time constant $(\mathrm{ms})$ & 5 & 1 & 3 \\
\hline
\end{tabular}

NEURON parameters that differed between units.

Table 3. Synaptic parameters

\begin{tabular}{lccccc}
\hline Parameter & Inp-Pyr & Inp-PV & Inp-SST & PV-Pyr & SST-Pyr \\
\hline Synaptic delay (ms) & 3 & 1 & 2 & 0.5 & 0.5 \\
U & 0.15 & 0.25 & 0.05 & 0.35 & 0.2 \\
$\tau_{\mathrm{d}}(\mathrm{ms})$ & 250 & 500 & 10 & 800 & 100 \\
$\tau_{\mathrm{f}}$ (ms) & 10 & 10 & 800 & 10 & 100 \\
\hline
\end{tabular}

NEURON parameters that differed between synapses.

less likely to be influenced by feedback circuitry (Rennaker et al., 2007). Furthermore, as we simulated a reduced circuit, the relatively large single synaptic currents reflect the near synchronous activation of multiple synapses (Bruno and Sakmann, 2006; DeWeese and Zador, 2006). This simplified circuit allowed a more tractable approach that was better constrained by the experimental data.

\section{Experimental design and statistical analysis}

For our analysis of the experimental dataset considered here (Natan et al., 2017), between-subjects variables included mouse genotype (PV-Cre or SST-Cre) and the temporal profile (adapting, steady, or facilitating). From five male PV-Cre mice, there were 225 adapting, 413 steady, and 45 facilitating unit-frequencies. From six male SST-Cre mice, there were 350 adapting, 412 steady, and 41 facilitating unit-frequencies. Withinsubjects variables included the serial position within each train of tone pips (1-8) and the presence or absence of light during optogenetic inactivation experiments (non-light vs light). To classify unit-frequencies based on their temporal profile (Fig. 1), and to assess firing latency (Fig. $5 C$ ), epochs containing light stimulation were excluded, yielding a minimum of 40 non-light epochs for each unit-frequency and serial position. Separating trials to assess the effect of prior light on the evoked activity at serial position 2 yielded a minimum of 20 epochs per condition. To classify unit-frequencies based on their temporal profile, Wilcoxon signed-rank tests (to test for the presence of evoked activity), linear regression models (to determine the slope of the temporal profile), and Wilcoxon rank-sum tests (to exclude potential interneurons) were employed. To compare latencies between temporal profiles, a one-way Kruskal-Wallis test was used, and the Dunn procedure was used to perform pairwise comparisons between the three temporal profiles. To test for the effect of prior light at tone 2, Wilcoxon signed-rank tests were employed.

For statistical analysis of the simulation results, we used the same statistical tests as for the experimental data. The between-subjects variable was the interneuron unit type that received simulated optogenetic inactivation (PV or SST). Within-subjects variables included the serial position within each train of tones (1-8) and presence or absence of simulated inactivation. For all simulations that were subject to statistical tests, 20 trials were performed for each condition.

\section{Results}

\section{A1 neurons exhibit distinct temporal profiles in response to repeated tones}

We first examined the temporal response profiles of A1 neurons to trains of eight consecutive tones presented at a $2.5 \mathrm{~Hz}$ (400 ms). From a total of 406 neurons across 11 animals (five PV-Cre and six SST-Cre), we identified a total of 1486 neuron- 


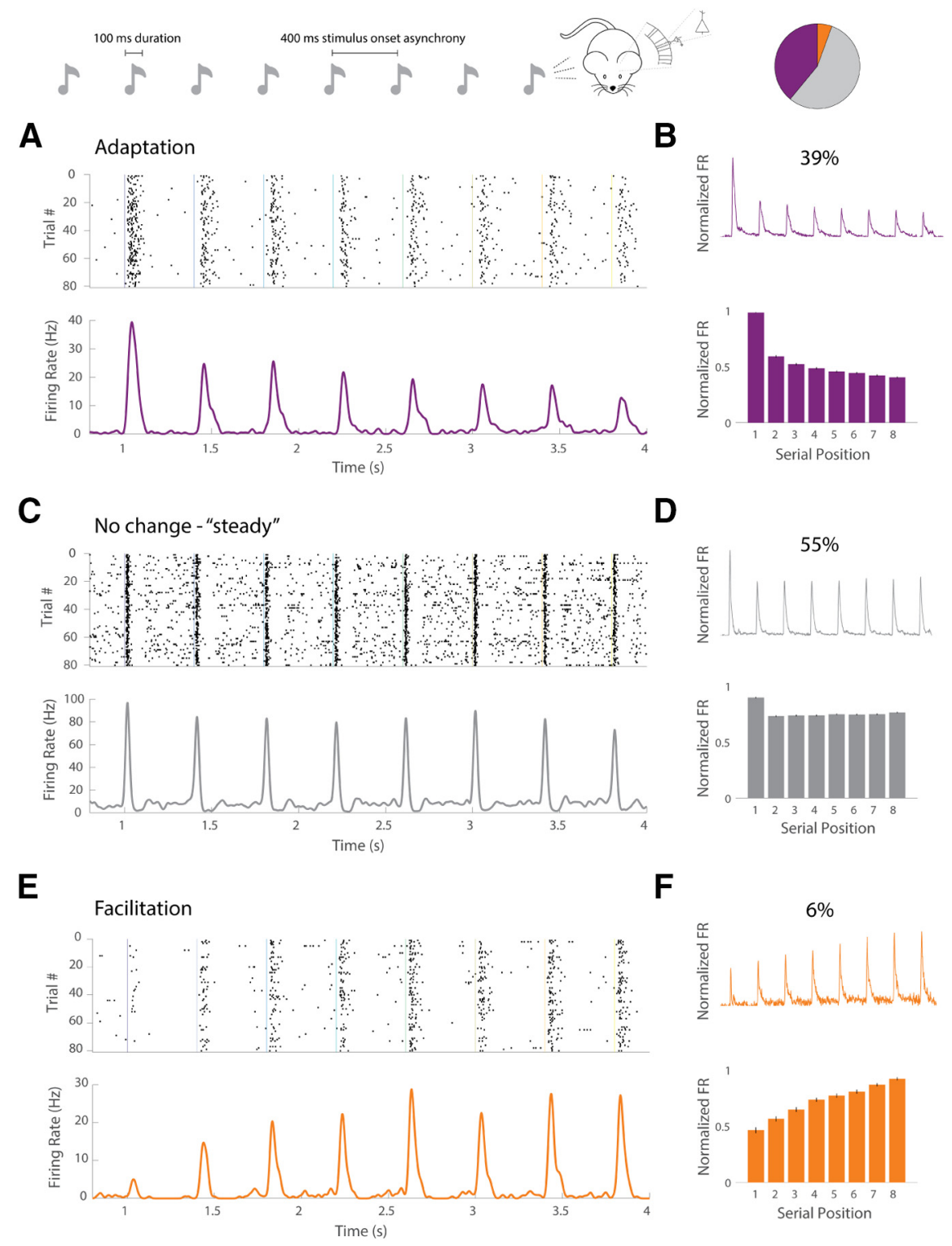

Figure 1. Single units in A1 exhibit diverse temporal profiles of evoked responses to sequences of repeated tones, including adaptation, no change, and facilitation. In the experiment, a train of eight consecutive repetitions of the same 100-ms pure tone stimulus was presented at a rate of $2.5 \mathrm{~Hz}$. The pie chart at top right shows the proportions of neuron-frequency pairs that exhibited adaptation (purple), stable (gray), or facilitation (orange). A, Spike raster (upper) and PSTH (lower) for a neuron-frequency pair that exhibits classical adaptation, in which sensory responses to the same physical stimulus decrease with repetition on short timescales. B, 39\% of neuron-frequencies with significant evoked activity were adapting. Population average normalized PSTH (upper) and bar plot of average normalized firing rate within 10-70 ms following tone onset by serial position (lower). C, Spike raster (upper) and PSTH (lower) for a neuron-frequency pair that exhibits no change or a stable firing rate over repetition. $\boldsymbol{D}, 55 \%$ of neuron-frequencies with significant evoked activity exhibited no change in firing rate over repetition. $\boldsymbol{E}$, Spike raster (upper) and PSTH (lower) for a neuron-frequency pair that exhibits facilitation, in which sensory responses to the same physical stimulus increase with repetition on short timescales. $F, 6 \%$ of neuron-frequencies with significant evoked activity were facilitating.

frequency pairs that exhibited a significant evoked response to either the first or last tone (see Materials and Methods). Based on the temporal profile of the number of spikes elicited in response to each of the eight tones in each train, we classified each neuron-frequency pair as adapting, stable, or facilitating (see Materials and Methods). Of these, 38.7\% were adapting, 55.5\% stable, and 5.8\% facilitating. Figure 1 displays sample raster plots of each type of temporal profile (Fig. $1 A, C, E$ ) and the population PSTHs across all neuronfrequency pairs within each category (Fig. $1 B, D, F)$. Consistent with previous studies these results confirm that while many neurons exhibit robust sensory adaptation, there is significant diversity in the temporal profile of the responses including neurons that exhibit robust facilitation.

\section{Model of a simple cortical microcircuit that incorporates STP}

The presence of adaptation or facilitation within cortical neurons establishes that responses are sensitive to temporal context, in other words, that there is a memory of recent stimulus history in the circuits. Early models suggested that one of the mechanisms underlying the sensitivity to temporal context is STP (Buonomano and Merzenich, 1995; Buonomano, 2000; Chung et al., 2002; Abbott and Regehr, 2004; Levy and Reyes, 2011; Rosenbaum et al., 2012; Motanis et al., 2018; Pérez and Merchant, 2018), and recent work has focused on the differential STP among different classes of inhibitory neurons as contributing to sensory adaptation (Natan et al., 2017; Phillips et al., 2017a). We thus developed a spike-based model of feedforward activation of $\mathrm{Pyr}$ neurons that incorporated the 
experimentally characterized STP of PV and SST neurons (Fig. 2).

Importantly, we used published, empirically-derived STP estimates for each of the five synapses in our Pyr-PV-SST microcircuit (Fig. 2A). We modeled mild depression at the Input $\rightarrow \mathrm{Pyr}$ synapse (Chung et al., 2002; Beierlein et al., 2003; Xu et al., 2007), moderate depression at the Input $\rightarrow$ PV synapse (Beierlein et al., 2003; Levy and Reyes, 2012; Takesian et al., 2013), strong facilitation at the Input $\rightarrow$ SST synapse (Tan et al., 2008; Takesian et al., 2013), strong depression at the PV $\rightarrow$ Pyr synapse (Gupta et al., 2000; Oswald and Reyes, 2011; Levy and Reyes, 2012), and stable synaptic strength at the SST $\rightarrow$ Pyr synapse (Silberberg and Markram, 2007; Takesian et al., 2010). STP parameters are shown in Table 3. Additionally, the intrinsic properties of the three neuron classes were based on aggregate estimates from the NeuroElectro project and select electrophysiological studies (Gibson et al., 1999; Tripathy et al., 2014). Using these units and synapses, we assembled a circuit with dual disynaptic feedforward inhibition from both PV and SST onto the Pyr unit and simulated the experimental protocol (Fig. 1).

\section{Model can account for all three temporal profiles by changing the inhibitory weights}

We next asked whether this simple model with empirically-based STP values at the five synapses could account for all three experimentally observed temporal profiles. Our goal was to determine whether the diversity of temporal profiles could be reproduced without changing the temporal dynamics of STP at each of the five synapses modeled here. Thus, the free parameters in the model were the five synaptic weights: Input $\rightarrow \mathrm{Pyr}$, Input $\rightarrow \mathrm{PV}$, Input $\rightarrow \mathrm{SST}, \mathrm{PV} \rightarrow \mathrm{Pyr}$, and SST $\rightarrow \mathrm{Pyr}$. We omitted higher order lateral connections, such as the SST $\rightarrow \mathrm{PV}$ connections, because these are unlikely contribute to the fast latency responses studied here (see Materials and Methods). The input weights onto all three neuron classes were constrained such that they elicited biologically reasonable firing rates in response to single tones in the absence of any inhibition. We thus anchored the input weights and focused on a parametric analysis of the weights of the $\mathrm{PV} \rightarrow \mathrm{Pyr}$ and SST $\rightarrow$ Pyr connections. As shown in Figure $2 B-D$, it is possible to transition between all three temporal profiles by changing only the $\mathrm{PV} \rightarrow \mathrm{Pyr}$ and SST $\rightarrow$ Pyr weights. Starting in a regime with weak inhibitory weights from both PV and SST, we observe a steady response profile (Fig. 2C). This is intuitive because in the absence of strong inhibition the temporal profile is primarily shaped by STP of the Input $\rightarrow \mathrm{Pyr}$ connection, which is weakly depressing. By increasing the strength of the SST $\rightarrow \mathrm{Pyr}$ connection, the system shifts to the adapting profile (Fig. 2B). Although early in the train SST generated inhibition is relatively weak, increasing the strength of the $\mathrm{PV} \rightarrow \mathrm{Pyr}$ connection shifts the profile from adapting to facilitating (Fig. $2 D$ ).

Figure 3 contrasts experimental (Fig. $3 A 1-C 1$ ) and simulated (Fig. 3A2-C2) examples of units from all three classes of temporal profiles. The unfilled bar plots display the model results of average evoked firing rates of the Pyr unit across twenty trials with independent noise currents provided to each unit. Importantly, the only parameters that were varied between the three simulated temporal profiles were the synaptic strengths (i.e., maximal conductances) of the SST $\rightarrow$ Pyr and the PV $\rightarrow$ Pyr synapses. All other parameters, including the STP parameters at each synapse, were the same across all simulations.

In order to quantify the robustness of the above results across different relative ratios of PV and SST inhibition we conducted a two-dimensional parameter search across the SST $\rightarrow \mathrm{Pyr}$ and
A
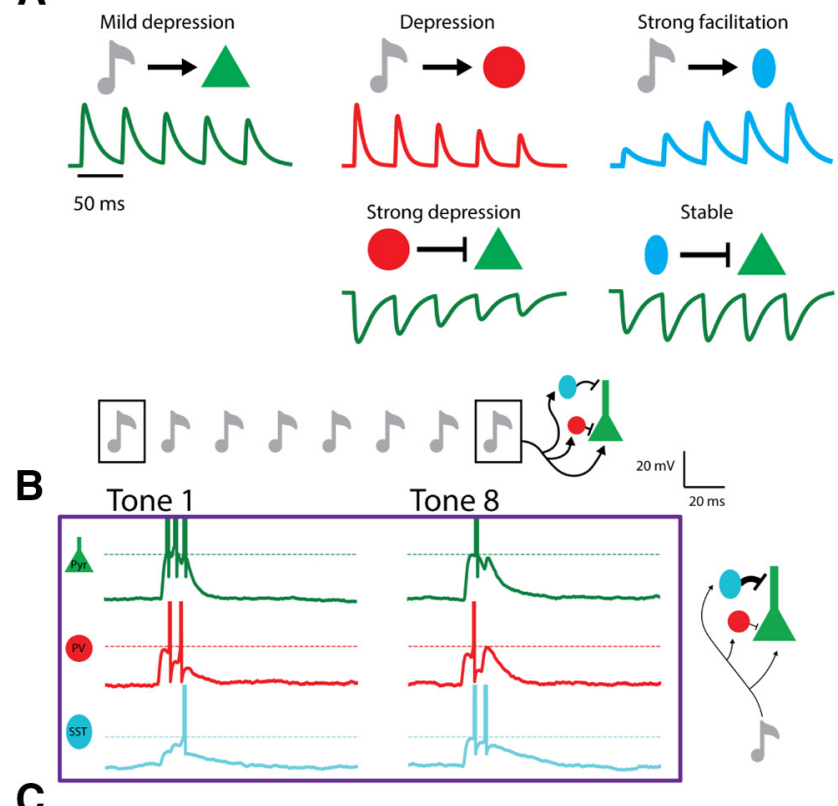

C

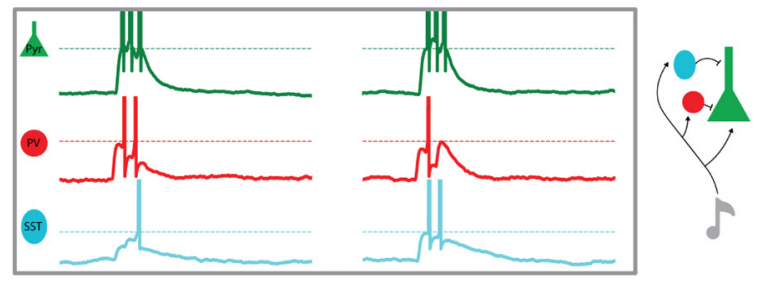

D

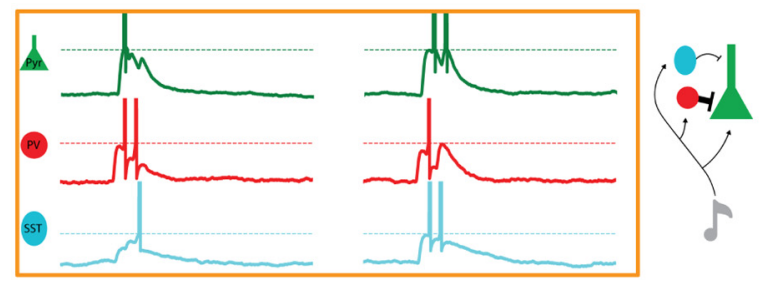

Figure 2. Spiking model of feedforward cortical microcircuit with empirically-based STP. $A$, Three distinct units were modeled to resemble cortical Pyr (green), fast-spiking PVexpressing interneurons (PV, red), and low-threshold-spiking SST-expressing interneurons (SST, cyan). The change in synaptic currents caused by repeated presynaptic spikes was governed by STP derived from experimental observations. $\boldsymbol{B}$, Single-unit membrane voltages from a model simulation of the experiment considered here (Natan et al., 2017). Because the SST $\rightarrow$ Pyr synapse is strong, Pyr unit spiking is suppressed during the eighth tone. $\boldsymbol{C}$, Single-unit membrane voltages when both the PV $\rightarrow$ Pyr and SST $\rightarrow$ Pyr synapses are relatively weak and balanced. Pyr unit spiking is relatively unaffected. $\boldsymbol{D}$, Single-unit membrane voltages when the PV $\rightarrow$ Pyr synapse is strong. Pyr unit spiking is strongly suppressed during the first tone but only weakly suppressed during the eighth tone, resulting in facilitation.

$\mathrm{PV} \rightarrow$ Pyr weights. At each combination of inhibitory weights, we quantified the average firing rate of the Pyr unit at each serial position across 20 trials. To quantify and visualize the temporal profiles, we regressed the Pyr unit firing rate against the serial position and took the slope as a quantitative index in which positive values indicated facilitation, negative values adaptation, and values near zero indicated a steady firing profile (Fig. $3 D$ ). These results confirm the robustness of the model across different inhibitory weights, and highlight the importance of the relative balance of PV and SST inhibition. One can see that the diagonal of the parametric analysis reveals mostly steady responses, indicating that it is not simply the absolute strength of PV or SST inputs that determines the temporal profile. For example, at $\mathrm{PV} \rightarrow \mathrm{Pyr}$ 

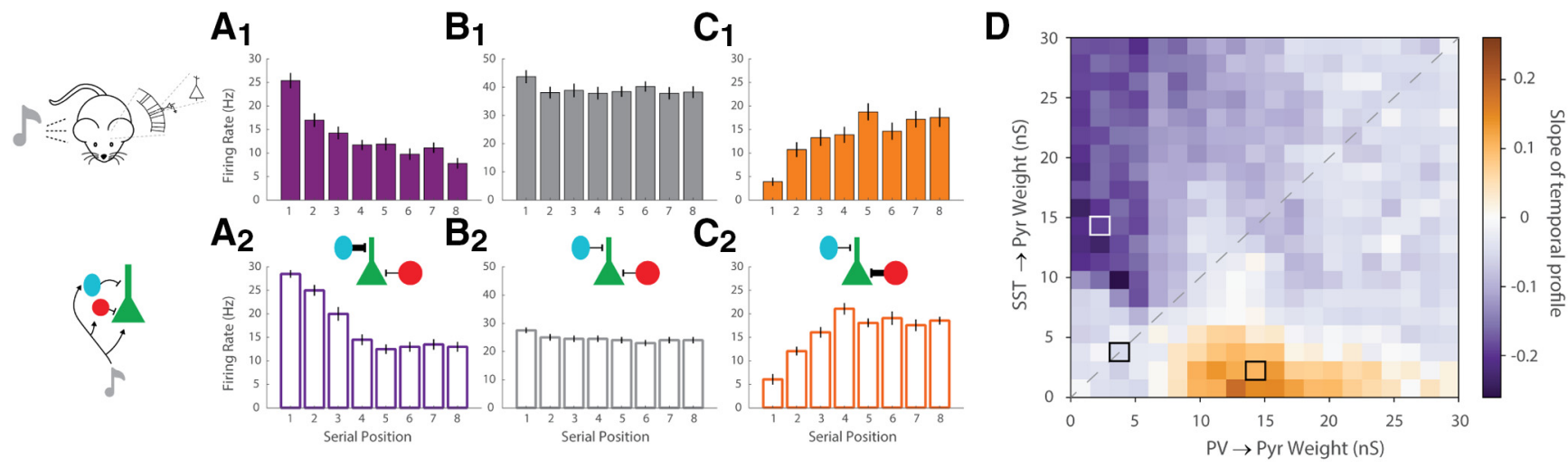

Figure 3. Model circuit with dual inhibition provided by PV and SOM units reproduced the experimentally observed adaptation $(\boldsymbol{A})$, steady responses $(\boldsymbol{B})$, and facilitation $(\boldsymbol{C})$ by only changing relative strength of PV/SST inhibition (while maintaining STP dynamics fixed). These three temporal profiles reflect three of the weight sets across a systematic parametric analysis of the $\mathrm{PV} \rightarrow$ Pyr and SST $\rightarrow$ Pyr weights (D). In A-C, the upper filled bar plot displays the average tone-evoked firing rate from 10 to $70 \mathrm{~ms}$ for an exemplary experimentally-observed neuron-frequency pair across a minimum of 40 trials, while the bottom, unfilled bar plot displays the average tone-evoked firing rate of the Pyr unit in the model across 20 trials with independent noise when synaptic weights were set as indicated by the inset diagrams and the outlined weight combinations shown in $\boldsymbol{D}$. All error lines indicate the SEM. $\boldsymbol{A}$, Exemple of an experimentally observed adapting response. A2, Simulated adapting Pyr unit. Bars indicate the mean firing rate evoked by a simulated sequence of "tones" based on their serial position. In this simulation, the SST $\rightarrow$ Pyr synapse was relatively strong, as indicated by the inset circuit diagram. B1, Experimental example of a steady neuron. B2, Simulated steady Pyr unit: both the PV $\rightarrow$ Pyr and SST $\rightarrow$ Pyr synapses were relatively weak and balanced, as indicated by the inset circuit diagram. C1, Experimental example of a facilitating Pyr neuron. C2, Simulated facilitating Pyr unit: the $\mathrm{PV} \rightarrow$ Pyr synapse was relatively strong, as indicated by the inset circuit diagram. D, Color-coded heatmap of the slope of the Pyr firing rate across serial positions (i.e., the temporal profile) as the weights PV $\rightarrow$ Pyr and SST $\rightarrow$ Pyr synapses were parametrically varied. More intensely purple squares reflect adaptation, while more intensely orange squares reflect facilitation.

weights of 10-15 nS, facilitating, steady, or depressing profiles can be observed, depending on the weight of the SST $\rightarrow \mathrm{Pyr}$ connection.

\section{STP acts via changes in spike latency}

The above results are driven by the dynamic shifts in E/I balance produced by STP. For example, facilitation of the Input $\rightarrow$ SST is responsible for the progressive decrease in Pyr firing in response to repeated tones. It is reasonable to assume that this modulation relies on an increase in the number of spikes in the SST neuron. A detailed analysis of the model, however, revealed a more complex mechanism. Specifically, much of the modulation of the firing rates of the Pyr neurons is not governed by the change in the spike number of inhibitory neurons, but by the shift in their firing latency.

To examine the importance of STP produced shifts in spike latency of inhibitory neurons in shaping Pyr profiles we first considered a circuit in the adaptation regime (Fig. $4 A$ ). As the Pyr unit firing rate decreases across serial positions, the first spike latency of the SST unit decreases in a correlated manner, because of shortterm facilitation of the Input $\rightarrow$ SST synapse. Although the SST unit's first spike latency and the Pyr unit's firing rate are simultaneously changing in a nonlinear manner, there was a strong linear correlation between the two $(r=0.99, p<0.001)$. In a circuit in the facilitation regime (Fig. $4 B$ ), the increase in Pyr unit firing rate across serial positions was correlated with an increase in first spike latency of the PV unit because of short-term depression of the Input $\rightarrow \mathrm{PV}$ synapse $(r=0.96, p<0.001)$; note, however, that the range of the latency shifts was narrower because of the relatively weak short-term depression at the Input $\rightarrow \mathrm{PV}$ connection. Importantly, we note that such a relationship will only be found for circuits with a single dominant source of inhibition and that in scenarios with weak or balanced sources of inhibition (which tend to exhibit steady temporal profiles) such a relationship will not hold.

The previous analyses suggest that decreases in the latency of SST spiking produced by short-term facilitation of the Input $\rightarrow$ SST synapse plays a role in the adaptation profile. But they do not demonstrate that the change in spike timing causes the change in Pyr unit firing rate within the model. To demonstrate a causal relationship, we developed an approach for artificially altering the timing of the inhibitory unit. We reran the simulations under the adapting regime, but replaced the spike times of the SST unit for tones 3-8 with the average spike times of the SST unit during tone 2 (Fig. $4 C$ ). Thus, we artificially "froze" the SST unit spike times and prevented the STP of the Input $\rightarrow$ SST synapses from naturally decreasing the tone-evoked latency of the SST neurons, while preserving the increase in spike number produced by STP during tones. This manipulation caused the Pyr unit to exhibit a steady firing rate across serial positions 2-8, eliminating the adaptation after tone 2 (Fig. $4 D$ ). Importantly, the firing rate of the SST unit in the trials where its spike timing was "frozen" was identical to its firing rate shown in Figure $3 A 2$. Thus, the temporal profile of adaptation can primarily be attributed to a progressive reduction in the tone-evoked spike latencies of the SST unit.

This result is consistent with previous experimental and computational data establishing that the temporal relationship between EPSPs and IPSPs onsets in the Pyr neurons plays a fundamental role in shaping neuronal response (Marder and Buonomano, 2004; Carvalho and Buonomano, 2009). The earlier the onset of the IPSP, the more effective it is at preventing the EPSP from driving the Pyr neuron to threshold. Our model predicts that the adaptation produced by progressive facilitation of the Input $\rightarrow$ SST synapse is not driven primarily by increased spiking of the SST neurons, but rather the progressive decrease in the latency of input-evoked SST spiking. It is relevant to stress that this latency effect would likely not be captured as well by firing rate models which are less well suited to pick up on the highly nonlinear interactions between shifts in EPSP/IPSP latencies and all-or-none spike generation (Pouille and Scanziani, 2001; Marder and Buonomano, 2004; Wilent and Contreras, 2005; Carvalho and Buonomano, 2009).

\section{Model correctly predicts longer response latencies under adaptation and facilitation}

Relative timing of the EPSCs and IPSCs onto the Pyr neurons is a critical determinant of the model. To be effective, inhibition 

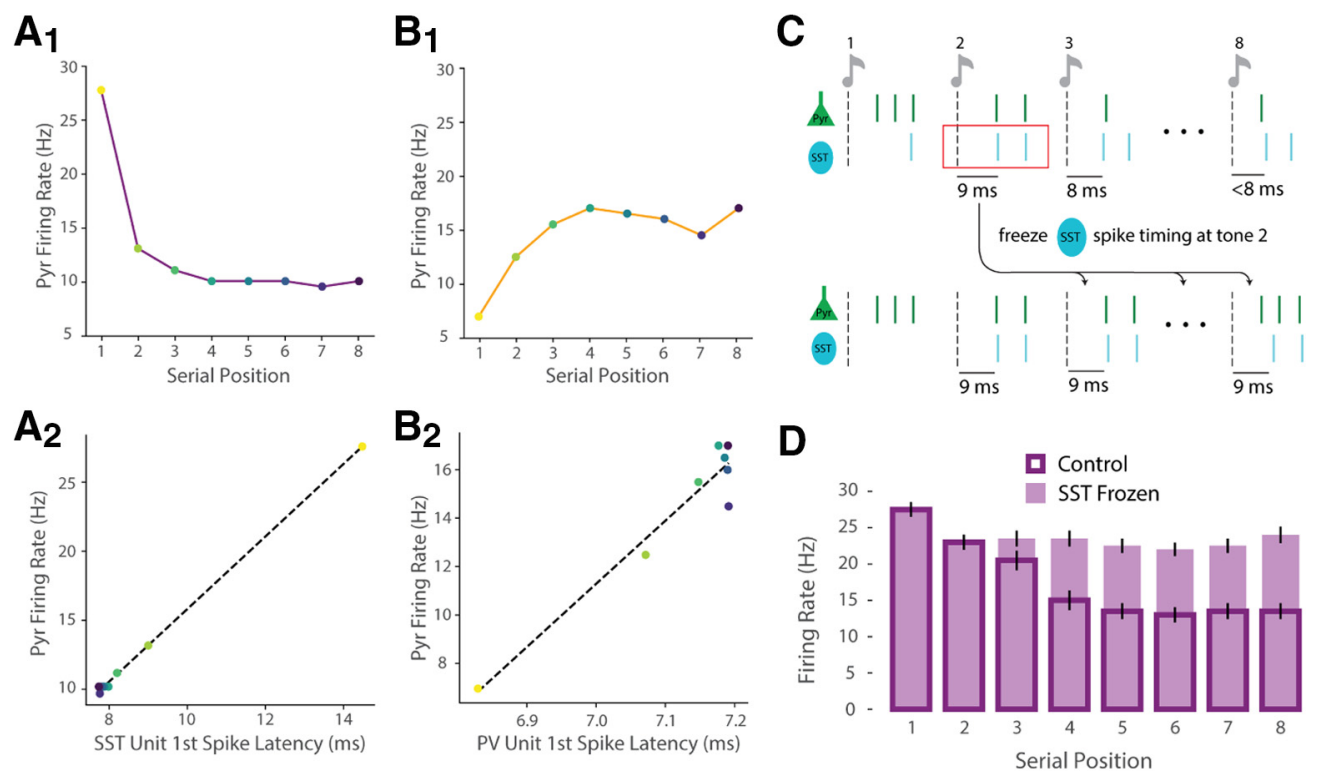

Figure 4. Temporal profiles are shaped by STP-driven changes in spike latency. A1, Decreasing firing rate in Pyr unit in a circuit in the adaptation regime across consecutive tones. $\mathbf{A 2}$, Relationship between firing rate in $\boldsymbol{A} \mathbf{1}$ and first spike latency of SST. Note that the color or each point corresponds to the serial position in $\boldsymbol{A}$. B1, Increasing firing rate in Pyr unit in a circuit in the facilitating regime across consecutive tones. Note the relatively narrow range of PV latency changes compared with SST. B2, Relationship between firing rate in A1 and first spike latency of PV. C, Starting from the model results in the adaptation weight regime, we recorded the average tone-evoked latency of SST spikes during the second tone. Then, we re-ran the simulation while artificially replacing the SST unit spiking on tones 3-8 to be the same as the average on tone 2 . D. When SST unit spiking was frozen at its latency during tone 2, adaptation was eliminated despite no change in the firing rate of the SST unit.

must arrive quickly enough to interact with excitatory inputs, preventing potential spiking. Based on this observation, we hypothesized that steady neurons do not undergo strong temporal-context modulation, because they often fire before the onset of inhibition. Thus, a key prediction of our model is that there should be a relationship between the input-evoked latency of the Pyr unit and the extent to which that unit's firing rate is decreased by inhibition. More specifically, we predicted that short-latency Pyr units should be less vulnerable to inhibition while still exhibiting mild forms of firing rate adaptation because of mild depression at the Input $\rightarrow$ Pyr synapse. In contrast, we predicted that longer latency Pyr units should be more strongly modulated by feedforward inhibition.

To test these predictions, we first measured the response latency of each neuron-frequency pair in the experimental data. We operationalized response latency as the time of the maximal peak in the PSTH in response to the first (adapting and stable responses) or final (facilitating responses) tone. Even within a temporal profile class response latency varied considerably, as evidenced by a plot of PSTHs sorted by latency (Fig. 5A,B). However, consistent with our prediction the mean latency of the steady group was significantly shorter than that of the adaptation and facilitation groups (Fig. 5C). Specifically, a one-way KruskalWallis test revealed a significant difference in response latency among the three classes $\left(\chi_{(2,1483)}^{2}=42.5, p<0.001\right)$, and post hoc Dunn tests revealed that both the adapting and facilitating classes of neuron-frequency pairs had significantly longer mean response latencies than the stable class (adapting vs stable, $Z=6.18$, $p<0.001$; facilitating vs stable, $Z=3.25, p<0.001$ ). However, the adapting and facilitating classes were not different from each other (adapting vs facilitating, $Z=-0.28, p=0.29$; Fig. $5 C$ ). This result provided support for the notion that Pyr cells that fire with a short latency, presumably because they receive strong excitatory synaptic inputs, undergo less temporal-context modulation because dynamic changes in IPSC strength are less effective in influencing Pyr neurons spiking generated by feed-forward activity. In other words, the inhibitory inputs onto these units are functionally weak because they are delayed relative to excitation.

Finally, because there was a large degree of variation in the response latency within a temporal profile, we considered whether the degree of adaptation or facilitation for a given unitfrequency was correlated with its response latency. We quantified the degree of adaptation or facilitation as the $t$-statistic resulting from the linear regression of tone-evoked spikes by the tone's serial position (see Materials and Methods). We found that for adapting unit-frequencies the degree of adaptation was mildly correlated with response latency $(r=-0.194, p<0.001)$ such that more strongly adapting unit-frequencies had longer evoked latencies. For facilitating unit-frequencies, this relationship was not significant.

\section{Model predicts a paradoxical decrease in firing caused by prior PV inactivation}

A long-standing challenge has been to establish a causal role for STP in temporal-context modulation. Interestingly, because of the model's reliance on STP to account for temporal-context effects, it led to a unique and counterintuitive prediction. Specifically, because STP is by definition dependent on the previous spike history on the time scale of tens-to-hundreds of milliseconds, preventing spikes evoked by a tone in one class of neurons should prevent STP and alter responses to subsequent tones. Indeed, as shown in Figure 6A, inhibition of PV neurons can sometimes not produce any effect during the optogenetically inhibited tone, but can alter the Pyr neuron response to a tone presented $400 \mathrm{~ms}$ later.

We first simulated optogenetic inhibition of PV and SST neurons by strongly hyperpolarizing either PV or SST model units from $100 \mathrm{~ms}$ before to $150 \mathrm{~ms}$ after the first tone presentation. Inhibitory inactivation of PV, but not SST, unit caused a historydependent effect on Pyr unit spiking under stable and facilitating weight regimes (Fig. 6B). More specifically, in the stable weight 


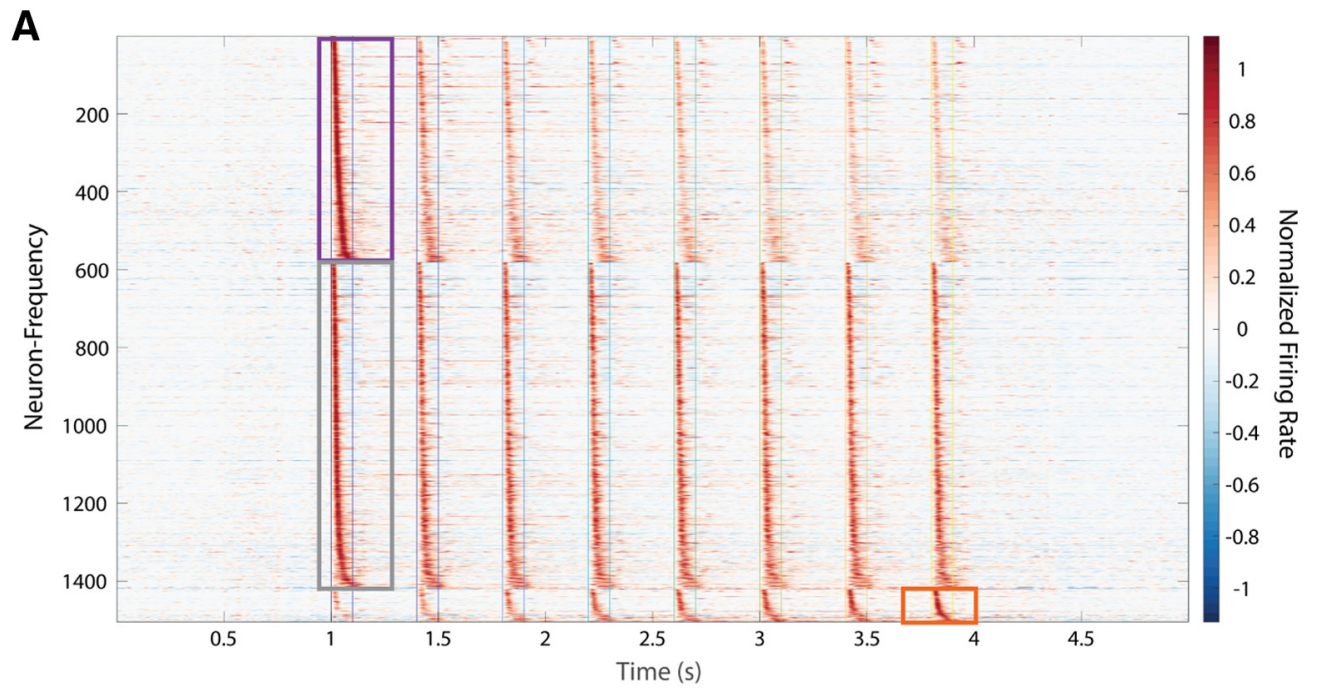

B

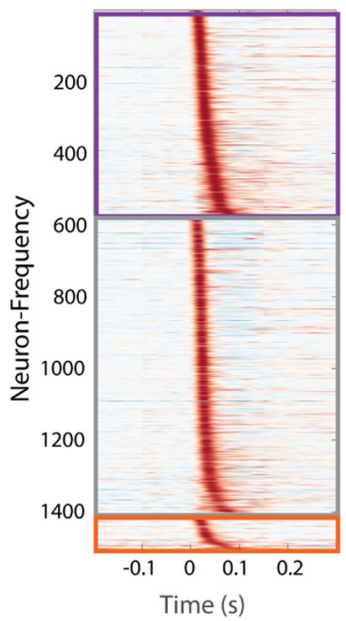

C

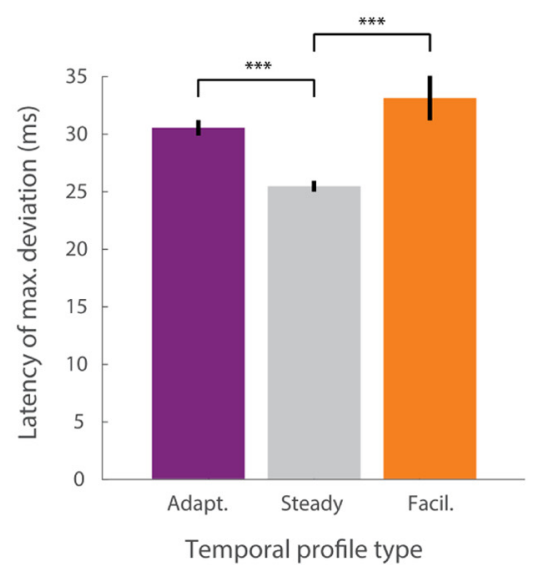

Figure 5. Evoked spike timing differs between temporal profile groups, according to model predictions. $\boldsymbol{A}$, Image plot of each neuron-frequency pair's PSTH, clustered by temporal profile (adapting, steady, facilitating) and sorted within each temporal profile by the latency of maximal evoked firing. Each row represents a single unit's average PSTH, expressed as the normalized deviation from baseline firing rate. Within adapting and steady temporal profiles, units are sorted by latency of maximum deviation to the first tone. Within the facilitating temporal profile, units are sorted by latency of maximum deviation to the eighth tone. $\boldsymbol{B}$, The same data in $\boldsymbol{A}$ re-plotted (see outlines) to show the distribution of average spike timing across trials for the time regions surrounding the peak response of that temporal profile. $\boldsymbol{C}$, Bar plot comparing the mean tone-evoked latencies within each temporal profile. Error lines indicate the SEM. There was a significant difference in response latency among the three classes $\left(\chi_{(2,1483)}^{2}=42.5, p<0.001\right)$. Both the adapting and facilitating classes of neuron-frequency pairs had significantly longer mean response latencies than the stable class (adapting vs stable, $Z=6.18, * * * p<0.001$; facilitating vs stable, $Z=3.25$, $* * * p<0.001$ ), but the adapting and facilitating classes were not different from each other (adapting vs facilitating, $Z=-0.28, p=0.29$ ).

regime, inactivating the $\mathrm{PV}$ unit during the first tone caused a decrease in the number of Pyr spikes evoked by the second tone (sign-rank $Z=-3.59 ; p<0.001$ ). We will refer to this as an " $n+1$ " effect because manipulation during one tone alters the response to the next tone in the absence of any further manipulation. A similar effect of slightly smaller magnitude was observed for the facilitating weight regime (sign-rank $Z=-3.42 ; p<$ $0.001)$. This $n+1$ effect was primarily a result of the STP profile of the PV $\rightarrow$ Pyr synapse (Fig. $2 B$ ). Because the PV unit normally spikes robustly during the first tone presentation, the $\mathrm{PV} \rightarrow \mathrm{Pyr}$ synapse is normally weakened during the second tone presentation because of its strong short-term depression, this depression normally counterbalances some of the some of the short-term depression of the EPSPs generated by the inputs. Hyperpolarizing and thus preventing the PV unit from spiking during the first tone effectively postpones its initial and strongest inhibitory effect until the second tone. Thus, following PV inactivation on the first tone, PV inhibition is stronger on the second tone, reducing the Pyr unit firing rate. Specifically, if spiking is inhibited during the first tone, the IPSP would not be depressed during the second tone, leading to a decrease in Pyr neurons spikes in response to the second tone. The $n+1$ effect in response to PV neuron inactivation was not observed in the adapting weight regime $(p>0.05)$, because it is dominated by SST inhibition (Fig. 6B, top panel). In contrast to PV inactivation, no effect of prior inactivation was observed for SST inactivation (all $p>0.05$; Fig. 6C) because the SST $\rightarrow$ Pyr synapse exhibited relatively little STP (furthermore the SST unit is relatively inactive during the first tone presentation).

Finally, we tested whether the $n+1$ effect was present in vivo (Fig. 6D,E). We contrasted the trials on which there was light inactivation during the first tone to trials in which there was no light inactivation during the first tone, and compared the number of spikes evoked by the second tone (note that there was no optogenetic inactivation during the second tone). In PV-Cre mice, there was a significant $n+1$ effect for stable neuron- 
A

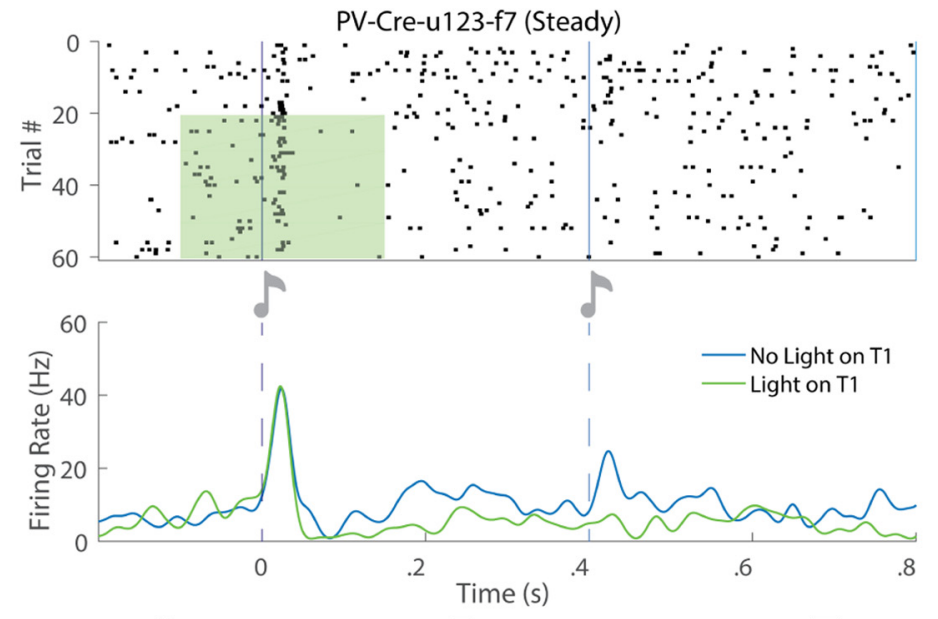

B
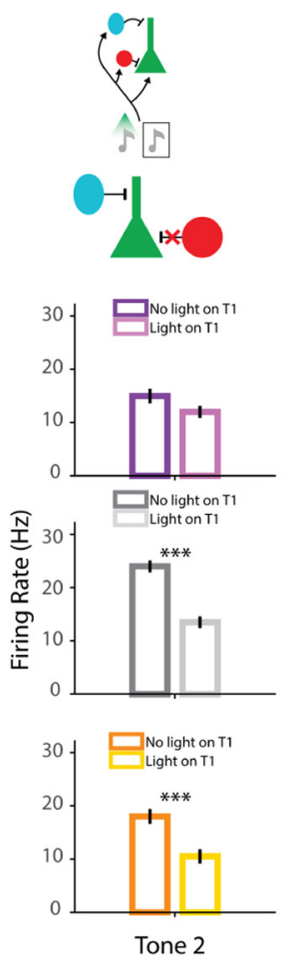

C
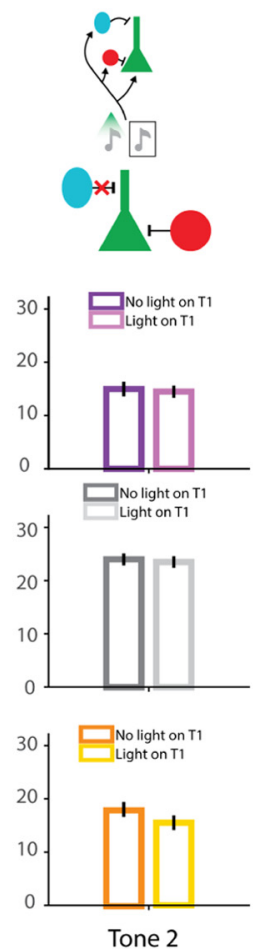

D
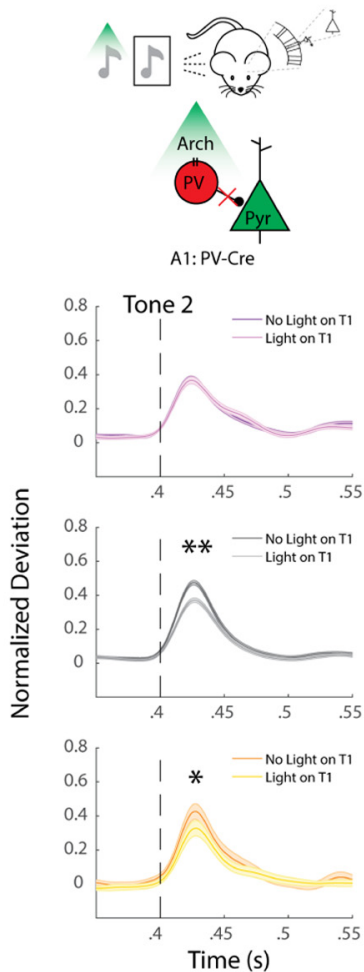

E
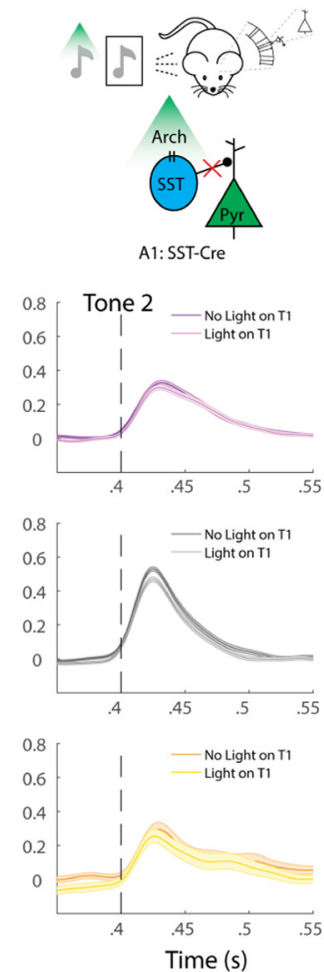

Figure 6. Simulated optogenetic inactivation of inhibitory units correctly predicts that PV interneuron inactivation during the first tone causes a decrease in the tone-evoked firing rate during the second tone only for steady and facilitating units. $A$, Example of an experimentally recorded Pyr neuron in which inactivation of PV interneurons during the first tone decreased the firing rate evoked by the second tone. In the spike raster (upper), the green rectangle indicates trials and window of optical stimulation. The superimposed line plots below display PSTHs (lower) separately for trials with and without optical stimulation during the first tone. $\boldsymbol{B}$, Effect of simulated PV inhibition on Pyr unit firing rates in adapting (purple), steady (gray), or facilitating (orange) regimes. Bars indicate the mean firing rate evoked by the second in a simulated pair of tones with and without simulated optogenetic inactivation of the PV unit during the first tone. Means were taken across 20 trials. Significant differences were found for the steady and facilitating weight regimes. C, Simulated SST inhibition on Pyr unit firing rate. SST inhibition during the first tone did not significantly alter Pyr firing in any of the three regimes $(p>0.05)$. $\boldsymbol{D}$, Experimentally observed effects of PV inhibition on Pyr firing to the subsequent tone, across all three temporal profile classes. Shaded regions indicate the SEM. Significant differences were found only for the steady and facilitating neuron-frequency pairs. $\boldsymbol{E}$, Mean normalized evoked firing rates of adapting (purple), steady (gray), or facilitating (orange) neuron-frequency pairs in response to SST inhibition. Consistent with the model predictions of the model no significant differences were observed in either class of temporal profiles $(p>0.05)$. $* p<0.05, * * p<0.01$, and $* * * p<0.001$.

frequency pairs (sign-rank $Z=-3.10 ; p=0.002$ ) and a significant but weaker prior light effect for facilitating neuron-frequency pairs (sign-rank $Z=-2.29 ; p=0.02$ ). Figure $6 \mathrm{~A}$ shows a raster plot and PSTH of a sample neuron that exhibited the $n+1$ effect, specifically inhibition of PV neurons during the first tone increased the number of spikes in response to the second tone. As in the computational model, the $n+1$ effect was not observed in the adapting group in response to PV inhibition $(p>0.05)$. Again, as in the model, there was no $n+1$ effect produced by
SST inactivation in either the adapting, stable, or facilitating groups (all $p>0.05$ ). These results provide a strong validation of a counterintuitive prediction of the model, and strong support for the role of STP in temporal-context modulation.

\section{Discussion}

A large body of experimental evidence has demonstrated that there is significant diversity in the temporal profile of the firing 
property of cortical neurons in response to repeated stimuli. However, the origin this diversity has remained largely unexplored. The current study identified a novel mechanism underlying temporal-context modulation of sensory stimuli as the source of the diversity of temporal response profiles. We found that a simple feedforward model that incorporated empiricallybased STP properties at five different synapses can account for the three experimentally observed temporal profiles by simply altering the relative weights of PV and SST inhibition. Critically the model generated two predictions that were experimentally tested and validated.

The first prediction was a relationship between temporal-profile class and tone-evoked spike latency. This prediction arises as a consequence of STP at the inhibitory branches the circuit (i.e., Input $\rightarrow$ PV $\rightarrow$ Pyr and Input $\rightarrow$ SST $\rightarrow$ Pyr), and, that for this STP to modulate Pyr firing the EPSCs and IPSCs onto a Pyr neuron must overlap in time. Thus, the strong temporal-context modulation in the adapting and facilitating groups suggest a broad temporal overlap between excitation and inhibition. In contrast, the relatively weak temporal context modulation in the stable populations, suggests that these neurons may fire too quickly to be strongly modulated by the inhibitory branch of the circuit which is delayed as a result of the additional synaptic step. This prediction was confirmed by demonstrating that the latency of the stable population was significantly shorter than that of the adapting and facilitating population (Fig. 5).

The second prediction of the model was that because temporal-context modulation is driven by changes in $\mathrm{E} / \mathrm{I}$ balance imposed by STP, that "blocking" STP should result in what we refer to as an $n+1$ effect. Specifically, that optogenetically inhibiting PV neurons should prevent potential STP at the PV $\rightarrow \mathrm{Pyr}$ synapse. If this STP is contributing to temporal-context modulation, then inhibition during tone $n$ should influence the Pyr neuron response at tone $n+1$. This novel prediction was experimentally confirmed by showing that inactivation of PV neurons at the first tone decreased firing of the Pyr neurons in response to the second tone (Fig. 6). In contrast, this effect was not observed by inhibiting the SST neurons, a result also predicted by the model because unlike the significant short-term depression at the $\mathrm{PV} \rightarrow \mathrm{Pyr}$, the $\mathrm{SST} \rightarrow \mathrm{Pyr}$ IPSP exhibits weak and inconsistent forms of STP. This result provides some of the best evidence to date that STP contributes to sensory adaptation.

\section{STP generates temporal-context modulation through spike rate and latency shifts}

It is increasingly recognized that PV and SST neurons fulfil distinct computational roles within cortical microcircuits (Atallah et al., 2012; Natan et al., 2015, 2017; Kato et al., 2017; Phillips et al., 2017a; Cardin, 2018). As both PV and SST neurons inhibit Pyr neurons, a crucial question pertains to which differences between PV and SST neurons underlie their different computational roles. Critical differences include distinct connectivity patterns (e.g., SST neurons primarily target Pyr dendrites), and intrinsic excitability (PV neurons are fast-spiking, and SST are low-threshold; Gupta et al., 2000; Oswald and Reyes, 2011). But one of the most robust differences between PV and SST neurons lies in their STP properties, specifically, excitatory inputs to PV neurons generally undergo short-term synaptic depression, while the excitatory synapses onto SST neurons exhibit dramatic facilitation (Reyes et al., 1998; Beierlein et al., 2003; Silberberg and Markram, 2007; Tan et al., 2008; Takesian et al., 2013). Using computational and experimental approaches we were able to quantify the contributions of inhibition not only to adaptation, but also to facilitation, and distinguish between firing rate and spike latency contributions. Our results provide for a novel differential role for PV and SST in temporal context modulation. Further, we find that the diversity of temporal response profiles in cortical neurons may be explained by the initial connectivity of local circuits, i.e., whether the dominant source of inhibition onto a Pyr neuron is tilted toward PV or SST neurons.

In contrast to previous models of how short-term plasticity may underlie temporal context modulation, which have relied on abstract or firing rate implementations, our spiking model emphasizes a key functional mechanism of STP. The dynamic decreases and increases in synaptic strength imposed by STP are not expressed solely by changing spike probability and rate, but by relatively small shifts in the latency of inhibitory neuron firing. That is, in the temporal relationship between the EPSCs and IPSCs impinging on the Pyr neurons. Indeed, previous experimental and computational results have demonstrated that both short-term and long-term synaptic plasticity can shape neural computations by modulating the race to threshold between EPSCs and IPSCs. For example, latency delays of a mere millisecond in inhibitory firing can convert a subthreshold excitatory input in to a suprathreshold input (Marder and Buonomano, 2004; Wilent and Contreras, 2005; Carvalho and Buonomano, 2009). Thus, the balance between excitation and inhibition is not simply governed by absolute synaptic strength but by the relative timing of EPSCs and IPSCs in the Pyr neurons. Thus, a further prediction that emerges from the model is that significant shifts in the latency of SST neurons should shorten in response to consecutive tones. And that it is possible that short-term facilitation onto SST neurons might not significantly increase SST firing rate, but still underlie adaptation through shifts in latency.

\section{Limitations of the model}

The model presented here is highly simplified in that it does not take into account the full complexity of cortical microcircuits, including the presence of recurrent excitation, and the connections between SST $\rightarrow \mathrm{PV}$ and $\mathrm{PV} \rightarrow \mathrm{SST}$ neurons (Pfeffer et al., 2013; Walker et al., 2016). Additionally, we focused on single neurons, rather than a large-scale population model (Park and Geffen, 2020). These simplifying assumptions allowed us to firmly ground the model in the empirical data and avoid making assumptions about the recurrent circuitry underlying cross-frequency interactions. Importantly, it is unlikely that that these higher-order circuit interactions significantly contribute to the short-latency Pyr neuron responses to single tones being studied here. Furthermore, there is evidence that sensory responses in anesthetized animals are driven primarily by feedforward circuitry, and that there is a significant increase in late-sustained responses in awake cortex (Wang et al., 2005; Moshitch et al., 2006; Rennaker et al., 2007). In order to further understand the cross-frequency suppression and facilitation effects, in the future, detailed population-level models will have to be developed. Toward this goal the current results highlight the importance of empirically defining the detailed microcircuit structure as latency differences of a few milliseconds can have a profound functional effect.

Critical to our objective of anchoring the model in the experimental data, are the assumptions related to the direction, magnitude, and temporal profile of STP at each synapse type. The model is largely robust to the changes in the empirically-derived values regarding the magnitude and times constants of STP. However, the presence of short-term facilitation at the Input $\rightarrow$ SST synapse, and depression at the $\mathrm{PV} \rightarrow \mathrm{Pyr}$ synapses are key to 
the presented results. And while there is significant variability in the experimental estimated of the STP parameters at the synapse classes simulated here, to the best of our knowledge all experimental data points to facilitation and depression of Input $\rightarrow$ SST and PV $\rightarrow$ Pyr synapses respectively (Reyes et al., 1998; Beierlein et al., 2003; Silberberg and Markram, 2007; Tan et al., 2008; Takesian et al., 2013; Pala and Petersen, 2015). Similarly, experimentally observed differences in the intrinsic properties of PV and SST neurons were also incorporated into the model. These included the time constants, spike threshold, spike adaptation, and the localization of inhibitory inputs onto the Pyr units. While these properties influence model behavior, they are not fundamental to obtaining the reported results.

\section{Predictions and conclusions}

In addition to the predictions tested here a number of additional predictions emerge from the current study, including: (1) the first-spike latency of SST neurons should decrease in response to consecutive tones; (2) adapting and facilitating neurons should receive strong SST and PV inhibition, respectively; and (3) that experience-dependent increases in responses to tone sequences (Kilgard and Merzenich, 2002; Engineer et al., 2008; Zhou et al., 2010) might be attributable to SST plasticity, e.g., long-term depression of SST IPSPs.

Overall our results suggest that differential PV and SST inhibition contributes to the diversity of temporal context profiles (Natan et al., 2015, 2017; Phillips et al., 2017a). Importantly the current results provide compelling evidence that STP is indeed a key mechanism driving temporal context modulation, by characterizing a novel $n+1$ effect that arises as a direct consequence of STP.

\section{References}

Abbott LF, Regehr WG (2004) Synaptic computation. Nature 431:796-803.

Ahissar E, Sosnik R, Bagdasarian K, Haidarliu S (2001) Temporal frequency of whisker movement. II. Laminar organization of cortical representations. J Neurophysiol 86:354-367.

Atallah BV, Bruns W, Carandini M, Scanziani M (2012) Parvalbuminexpressing interneurons linearly transform cortical responses to visual stimuli. Neuron 73:159-170.

Beierlein M, Gibson JR, Connors BW (2003) Two dynamically distinct inhibitory networks in layer 4 of the neocortex. J Neurophysiol 90:2987-3000.

Brosch M, Schreiner CE (1997) Time course of forward masking tuning curves in cat primary auditory cortex. J Neurophysiol 77:923-943.

Brosch M, Schreiner CE (2000) Sequence sensitivity of neurons in cat primary auditory cortex. Cereb Cortex 10:1155-1167.

Bruno RM, Sakmann B (2006) Cortex is driven by weak but synchronously active thalamocortical synapses. Science 312:1622-1627.

Buonomano DV (2000) Decoding temporal information: a model based on short-term synaptic plasticity. J Neurosci 20:1129-1141.

Buonomano DV, Merzenich MM (1995) Temporal information transformed into a spatial code by a neural network with realistic properties. Science 267:1028-1030.

Cardin JA (2018) Inhibitory interneurons regulate temporal precision and correlations in cortical circuits. Trends Neurosci 41:689-700.

Carvalho TP, Buonomano DV (2009) Differential effects of excitatory and inhibitory plasticity on synaptically driven neuronal input-output functions. Neuron 61:774-785.

Carvalho TP, Buonomano DV (2011) A novel learning rule for long-term plasticity of short-term synaptic plasticity enhances temporal processing. Front Integr Neurosci 5:20.

Chung S, Li X, Nelson SB (2002) Short-term depression at thalamocortical synapses contributes to rapid adaptation of cortical sensory responses in vivo. Neuron 34:437-446.

Destexhe A, Mainen ZF, Sejnowski TJ (1994) An efficient method for computing synaptic conductances based on a kinetic model of receptor binding. Neural Comput 6:14-18.
DeWeese MR, Zador AM (2006) Non-Gaussian membrane potential dynamics imply sparse, synchronous activity in auditory cortex. J Neurosci 26:12206-12218.

Douglas RJ, Martin KAC (2007) Mapping the matrix: the ways of neocortex. Neuron 56:226-238.

Doupe AJ, Kuhl PK (1999) Birdsong and human speech: common themes and mechanisms. Annu Rev Neurosci 22:567-631.

Engineer CT, Perez CA, Chen YH, Carraway RS, Reed AC, Shetake JA, Jakkamsetti V, Chang KQ, Kilgard MP (2008) Cortical activity patterns predict speech discrimination ability. Nat Neurosci 11:603-608.

Fortune ES, Rose GJ (2001) Short-term synaptic plasticity as a temporal filter. Trends Neurosci 24:381-385.

Gibson JR, Beierlein M, Connors BW (1999) Two networks of electrically coupled inhibitory neurons in neocortex. Nature 402:75-79.

Goudar V, Buonomano DV (2015) A model of order-selectivity based on dynamic changes in the balance of excitation and inhibition produced by short-term synaptic plasticity. J Neurophysiol 113:509-523.

Gupta A, Wang Y, Markram H (2000) Organizing principles for a diversity of GABAergic interneurons and synapses in the neocortex. Science 287:273-278.

Gutnisky DA, Dragoi V (2008) Adaptive coding of visual information in neural populations. Nature 452:220-224.

Hines ML, Carnevale NT (1997) The NEURON simulation environment. Neural Comput 9:1179-1209.

Kato HK, Asinof SK, Isaacson JS (2017) Network-level control of frequency tuning in auditory cortex. Neuron 95:412-423.e4.

Katz Y, Heiss JE, Lampl I (2006) Cross-whisker adaptation of neurons in the rat barrel cortex. J Neurosci 26:13363-13372.

Kilgard MP, Merzenich MM (2002) Order-sensitive plasticity in adult primary auditory cortex. Proc Natl Acad Sci USA 99:3205-3209.

Klampfl S, David SV, Yin P, Shamma SA, Maass W (2012) A quantitative analysis of information about past and present stimuli encoded by spikes of A1 neurons. J Neurophysiol 108:1366-1380.

Klump GM, Gerhardt HC (1987) Use of non-arbitrary acoustic criteria in mate choice by female gray tree frogs. Nature 326:286-288.

Kohn A (2007) Visual adaptation: physiology, mechanisms, and functional benefits. J Neurophysiol 97:3155-3164.

Levy RB, Reyes AD (2011) Coexistence of Lateral and Co-Tuned Inhibitory Configurations in Cortical Networks. PLOS Comput Biol 7:e1002161.

Levy RB, Reyes AD (2012) Spatial profile of excitatory and inhibitory synaptic connectivity in mouse primary auditory cortex. J Neurosci 32:56095619.

Marder CP, Buonomano DV (2004) Timing and balance of inhibition enhance the effect of long-term potentiation on cell firing. J Neurosci 24:8873-8884

Markram H, Wang Y, Tsodyks M (1998) Differential signaling via the same axon of neocortical pyramidal neurons. Proc Natl Acad Sci USA 95:5323-5328.

Moshitch D, Las L, Ulanovsky N, Bar-Yosef O, Nelken I (2006) Responses of neurons in primary auditory cortex (A1) to pure tones in the halothaneanesthetized cat. J Neurophysiol 95:3756-3769.

Motanis H, Seay MJ, Buonomano DV (2018) Short-term synaptic plasticity as a mechanism for sensory timing. Trends Neurosci 41:701-711.

Natan RG, Briguglio JJ, Mwilambwe-Tshilobo L, Jones SI, Aizenberg M, Goldberg EM, Geffen MN (2015) Complementary control of sensory adaptation by two types of cortical interneurons. Elife 4:e09868.

Natan RG, Rao W, Geffen MN (2017) Cortical interneurons differentially shape frequency tuning following adaptation. Cell Rep 21:878-890.

Nelken I, Chechik G (2007) Information theory in auditory research. Hear Res 229:94-105.

Ohzawa I, Sclar G, Freeman RD (1982) Contrast gain control in the cat visual cortex. Nature 298:266-268.

Oswald AM, Reyes AD (2008) Maturation of intrinsic and synaptic properties of layer $2 / 3$ pyramidal neurons in mouse auditory cortex. J Neurophysiol 99:2998-3008.

Oswald AM, Reyes AD (2011) Development of inhibitory timescales in auditory cortex. Cereb Cortex 21:1351-1361.

Pala A, Petersen CC (2015) In vivo measurement of cell-type-specific synaptic connectivity and synaptic transmission in layer $2 / 3$ mouse barrel cortex. Neuron 85:68-75.

Park Y, Geffen MN (2020) A circuit model of auditory cortex. PLoS Comput Biol 16:e1008016. 
Pérez O, Merchant H (2018) The synaptic properties of cells define the hallmarks of interval timing in a recurrent neural network. J Neurosci 38:4186-4199.

Pfeffer CK, Xue M, He M, Huang ZJ, Scanziani M (2013) Inhibition of inhibition in visual cortex: the logic of connections between molecularly distinct interneurons. Nat Neurosci 16:1068-1076.

Phillips DP, Hall SE, Hollett JL (1989) Repetition rate and signal level effects on neuronal responses to brief tone pulses in cat auditory cortex. J Acoust Soc Am 85:2537-2549.

Phillips EAK, Schreiner CE, Hasenstaub AR (2017a) Cortical interneurons differentially regulate the effects of acoustic context. Cell Rep 20:771-778.

Phillips EAK, Schreiner CE, Hasenstaub AR (2017b) Diverse effects of stimulus history in waking mouse auditory cortex. J Neurophysiol 118:13761393.

Pouille F, Scanziani M (2001) Enforcement of temporal fidelity in pyramidal cells by somatic feed-forward inhibition. Science 293:1159-1163.

Rennaker RL, Carey HL, Anderson SE, Sloan AM, Kilgard MP (2007) Anesthesia suppresses nonsynchronous responses to repetitive broadband stimuli. Neuroscience 145:357-369.

Reyes A, Sakmann B (1999) Developmental switch in the short-term modification of unitary EPSPs evoked in layer 2/3/and layer 5 pyramidal neurons of rat neocortex. J Neurosci 19:3827-3835.

Reyes A, Lujan R, Rozov A, Burnashev N, Somogyi P, Sakmann B (1998) Target-cell-specific facilitation and depression in neocortical circuits. Nat Neurosci 1:279-285.

Rose G, Leary C, Edwards C (2011) Interval-counting neurons in the anuran auditory midbrain: factors underlying diversity of interval tuning. J Comp Physiol A Neuroethol Sens Neural Behav Physiol 197:97-108.

Rosenbaum R, Rubin J, Doiron B (2012) Short term synaptic depression imposes a frequency dependent filter on synaptic information transfer. PLoS Comput Biol 8:e1002557.

Saberi K, Perrott DR (1999) Cognitive restoration of reversed speech. Nature 398:760-760.

Sadagopan S, Wang X (2009) Nonlinear spectrotemporal interactions underlying selectivity for complex sounds in auditory cortex. J Neurosci 29:11192-11202.

Shu ZJ, Swindale NV, Cynader MS (1993) Spectral motion produces an auditory after-effect. Nature 364:721-723
Silberberg G, Markram H (2007) Disynaptic inhibition between neocortical pyramidal cells mediated by Martinotti cells. Neuron 53:735-746.

Solomon SG, Kohn A (2014) Moving sensory adaptation beyond suppressive effects in single neurons. Curr Biol 24:R1012-R1022.

Takesian AE, Kotak VC, Sanes DH (2010) Presynaptic GABA(B) receptors regulate experience-dependent development of inhibitory short-term plasticity. J Neurosci 30:2716-2727.

Takesian AE, Kotak VC, Sharma N, Sanes DH (2013) Hearing loss differentially affects thalamic drive to two cortical interneuron subtypes. J Neurophysiol 110:999-1008.

Tallal P (2004) Improving language and literacy is a matter of time. Nat Rev Neurosci 5:721-728.

Tan Z, Hu H, Huang ZJ, Agmon A (2008) Robust but delayed thalamocortical activation of dendritic-targeting inhibitory interneurons. Proc Natl Acad Sci USA 105:2187-2192.

Tripathy SJ, Savitskaya J, Burton SD, Urban NN, Gerkin RC (2014) NeuroElectro: a window to the world's neuron electrophysiology data. Front Neuroinformatics 8:40.

Walker F, Möck M, Feyerabend M, Guy J, Wagener RJ, Schubert D, Staiger JF, Witte M (2016) Parvalbumin- and vasoactive intestinal polypeptideexpressing neocortical interneurons impose differential inhibition on Martinotti cells. Nat Commun 7:13664.

Wang X, Lu T, Snider RK, Liang L (2005) Sustained firing in auditory cortex evoked by preferred stimuli. Nature 435:341-346.

Wehr M, Zador AM (2005) Synaptic mechanisms of forward suppression in rat auditory cortex. Neuron 47:437-445.

Wilent WB, Contreras D (2005) Dynamics of excitation and inhibition underlying stimulus selectivity in rat somatosensory cortex. Nat Neurosci 8:1364-1370

Wilson DA (2000) Odor specificity of habituation in the rat anterior piriform cortex. J Neurophysiol 83:139-145.

Xu H, Kotak VC, Sanes DH (2007) Conductive hearing loss disrupts synaptic and spike adaptation in developing auditory cortex. J Neurosci 27:94179426.

Zhou X, de Villers-Sidani É, Panizzutti R, Merzenich MM (2010) Successivesignal biasing for a learned sound sequence. Proc Natl Acad Sci USA 107:14839-14844 